\title{
Conspectus on Indian Gymnacranthera and Myristica
}

\author{
D. Banik ${ }^{1 *}$, P.P. Bora1, V. Sampath Kumar ${ }^{2}$ and R.L. Bezbaruah ${ }^{1}$ \\ ${ }^{1}$ CSIR-North East Institute of Science and Technology (NEIST), Jorhat - 785006, Assam, India. \\ ${ }^{2}$ Central National Herbarium, Botanical Survey of India, P.O. Botanic Garden, Howrah - 711103, \\ West Bengal, India. \\ *E-mail: banikdipanwita@yahoo.com
}

\begin{abstract}
Since, Sir J.D. Hooker's the Flora of British India, no comprehensive work on the family Myristicaceae R. Br., nom. cons. in India has been made but the family was treated with confusion in several regional floras, which were devoid of updated nomenclature and citation of type specimens. Recent work on Endocomia, Horsfieldia and Knema revealed that there are 5 genera and 18 species (including 1 cultivated species) and 4 subspecies distributed in India, of which nearly $45 \%$ of the taxa are endemic to the country. Therefore, the present taxonomic concept of the genera Gymnacranthera and Myristica in India became urgent and essential. The present study found that the genus Gymnacranthera is represented with 1 endemic species and Myristica with 5 species and 2 subspecies, of which 6 taxa are endemic to India. These taxa are cited with updated nomenclature, brief description, distribution, flowering and fruiting period, vernacular names, uses, notes and specimens examined. A key to the taxa are provided along with illustration and a photo plate picturing live specimens of 4 species to facilitate identification of the taxa.
\end{abstract}

Keywords: Gymnacranthera, India, Myristica, Myristicaceae

\section{Introduction}

A few monographs and revisionary works on the family Myristicaceae in British Indian territory (including Nepal, Bhutan, Bangladesh, Sri Lanka, Myanmar, Singapore and Peninsular Malaysia) were published by Hooker \& Thomson (1855), de Candolle (1856), Bentham \& Hooker (1883), Hooker (1886) and King (1890). Until monograph by Warburg (1897), all Myristicaceae species were known under the sole genus Myristica. Myristica fragrans Houtt., the nutmeg of commerce is the type of the genus Myristica Gronov. (Gronovius, 1755; Ludwig, 1760; Houttuyn, 1773). Altogether nine species under the genus were treated with brief descriptions by Roxburgh (1832), but the identity of species were difficult to determinate. Revisionary works on certain genera of the family concerning also India were published by Sinclair (1958, 1961, 1968, 1975) and de Wilde (1984a,b, 1997). Most recent work on the genera Endocomia, Horsfieldia and Knema in India by Banik \& Bora $(2016 a, b)$ created the need for the present taxonomic account of the genera Gymnacranthera and Myristica in India as hitherto no comprehensive work has been published on these genera in India since Hooker's the Flora of British India (1890). To solve these deficiencies, the present study was carried out and taxonomic account on these two genera are prepared for the first time for the whole of India, based on critical study of earlier revisionary works, protologues, type specimens and other specimens deposited in various regional, national and international herbaria. The work was initiated during 1999-2000 under 'Flora of India' Project for the 'Flora of West Bengal' at Central National Herbarium, Botanical Survey of India by the first author. Herbarium consultations including those of TBGT, K, BM and E were carried out later as a part of SERB, DST funded project since 2012 (Project file no. SR/FT/LS-39/2011).

\section{Methods}

Standard taxonomic procedures were followed. The protologues of accepted names and synonyms were consulted along with major and minor revisionary and monographic and other relevant literature. Types and herbarium specimens in CAL, TBGT, K, BM and E were studied. Data on flowering and fruiting period, habitat, vernacular names and uses of various parts of different species were collected from their area of occurrence, herbarium labels and existing literature. Herbarium codes (acronyms) are followed as in Holmgren et al. (1990). Abbreviations of the titles of the books not available at International Plant Names Index (www.ipni.org) are abbreviated in the manuscript 
as given below. The Useful Plants of India (Drury, 1858) as Drury, Useful Pl. India; Flowering Plants of the Western Ghats (Nayar et al., 2014) as T.S. Nayar et al., Fl. Pl. Western Ghats.

\section{Results}

During the study period, nearly 150 herbarium specimens of the genera Gymnacranthera and Myristica were studied and their identity was confirmed or changed. The identity of 5 genera and 18 species (including 1 cultivated) and 4 subspecies as occurring in India was confirmed. Out of these 22 taxa, 10 are endemic to India, resulting in nearly $45 \%$ endemism. The present study found that the genus Gymnacranthera is represented with one endemic species and Myristica with five species and two subspecies, of which six taxa are endemic to India. The present study also found that the species, M. fragrans Houtt. is cultivated in India. The paper provides a comprehensive taxonomic account on Gymnacranthera and Myristica in India with updated nomenclature of all taxa along with types, key to species, flowering and fruiting period, habitat, distribution, vernacular names, uses and notes. A photo plate comprising four species and one illustration are also provided for their easy identification.

\section{Key to genera}

1. Perianth splitting nearly to the base at anthesis; lobes pubescent inside ... 1. Gymnacranthera

1. Perianth splitting nearly to $1 / 3$ rd to $2 / 3$ rds at anthesis; lobes glabrous inside .. 2. Myristica

\section{Gymnacranthera (A. DC.) Warb.}

Gymnacranthera (A. DC.) Warb., Ber. Pharm. Ges. 2: 227. 1892; Ber. Deutsch. Bot. Ges. 13: 94.1896 \& Nova Acta Acad. Caes. Leop.-Carol. German. Nat. Cur. 68: 131. 1897; W.J. de Wilde, Fl. Males., Ser. 1, Spermat. 14: 40. 2000; R.T.A. Schouten, Blumea 31: 451. 1986. Myristica sect. Gymnacranthera A. DC., Ann. Sci. Nat., Bot. Sér. 4, 4: 31. 1855 \& Prodr. 14, 1: 200. 1856; Miq., Fl. Ned. Ind. 1(2): 63. 1858; King, Ann. Roy. Bot. Gard. Calcutta 3: 304. 1891. Type: Gymnacranthera paniculata (A. DC.) Warb.

Trees, dioecious, tall. Leaves lanceolate, whitish beneath. Lobes involute at anthesis, pubescent inside. Seeds blunt at ends.

Distribution: Nearly 7 species, distributed from southern India to eastern side of New Guinea; only 1 species is distributed in India.
1. Gymnacranthera canarica (Bedd. ex King) Warb., Nova Acta Acad. Caes. Leop.-Carol. German. Nat. Cur. 68: 368, t. 20, 1-5. 1897; R.T.A. Schouten, Blumea 31: 461. 1986; T.S. Nayar et al., Fl. Pl. Western Ghats 1: 663. 2014. Myristica canarica Bedd. ex King, Ann. Roy. Bot. Gard. Calcutta 3: 307, t. 138. 1891. Type: INDIA, South India, Tamil Nadu, Tinnevelley hills, December 1879 (12/79), Beddome 6724 (lectotype, K!) (vide Schouten, 1986, 1.c.).

Myristica farquhariana auct. non Hook.f. \& Thomson, 1855, p.p.: quoad Hohenacker 541; A. DC., Prodr. 14(1): 200. 1856, p.p.; Miq., Fl. Ned. Ind. 1(2): 63. 1859; Bedd., Fl. Sylv. S. India: t. 270. 1869, p.p.; Hook.f., Fl. Brit. India 5: 108. 1886, p.p.; J. Sinclair, Gard. Bull. Singapore 17: 96, 113. 1958, p.p...................... Fig. 1, $2 \mathbf{f}$ Vernacular Names: Kannada: Hedehagalu, Pindi, Pindikai; Malayalam: Pintikkaya, Undai panu; Tamil: Uudippanu.

Tree, dioecious with knee roots; leaves lanceolate, 9-22 × 3.5-7.5 cm, acute-obtuse at base, entire at margins, acuminate-cuspidate at apex. Inflorescence axillary, paniculate, branched; cymule many-flowered, flowers at different stages of development, puberulous; male flowers globose-ellipsoid, valves 3 or 4 , splitting the perianth in anthesis to the base; lobes involute at anthesis, puberulous inside; synandrium ellipsoid, androphore c. $0.5 \mathrm{~mm}$ long; stamens 5-15; anthers linear; slightly unequal, adnate to their back up to $1 \mathrm{~mm}$ from base, apical $1 \mathrm{~mm}$ free, incurved-spreading at anthesis, extrorse; female inflorescences racemose-paniculate, contracted; flower buds ellipsoid-obovoid, 2-3 × 1-1.5 mm, villous inside, tomentose outside, brown, scented; stigma sessile, 2-lobed; fruits globose with pointed apex, 2.4-2.6 cm; pericarp 1.5-2 mm thick, wrinkled, glabrous-puberulous, brown; aril laciniated almost up to base with linear pointed structure, yellow; seeds globose, blunt at ends with a persistent collar-like structure at base.

Flowering: December-April; Fruiting: June-July.

Habitat: Grows along streams in wet evergreen forests, to $750 \mathrm{~m}$ elevation. Common in Myristica swamps.

Distribution: India (Karnataka, Kerala and Tamil $\mathrm{Nadu}$ ). Endemic.

IUCN Threat Status: Vulnerable B1+2c, D2 ver. 2.3 (IUCN, 2017-1).

Uses: Candles and soaps are made from seeds (CSIR, 1956). 


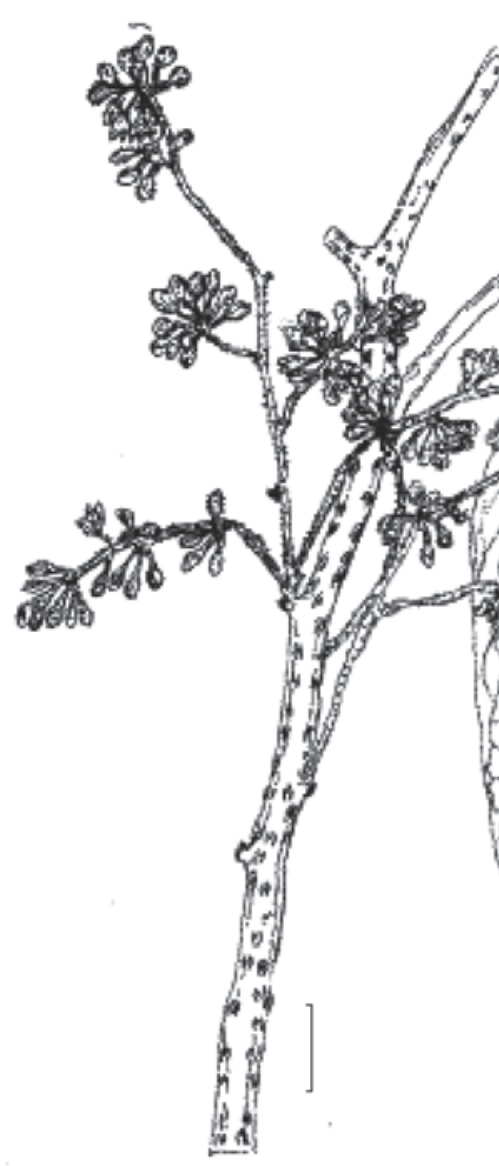

a
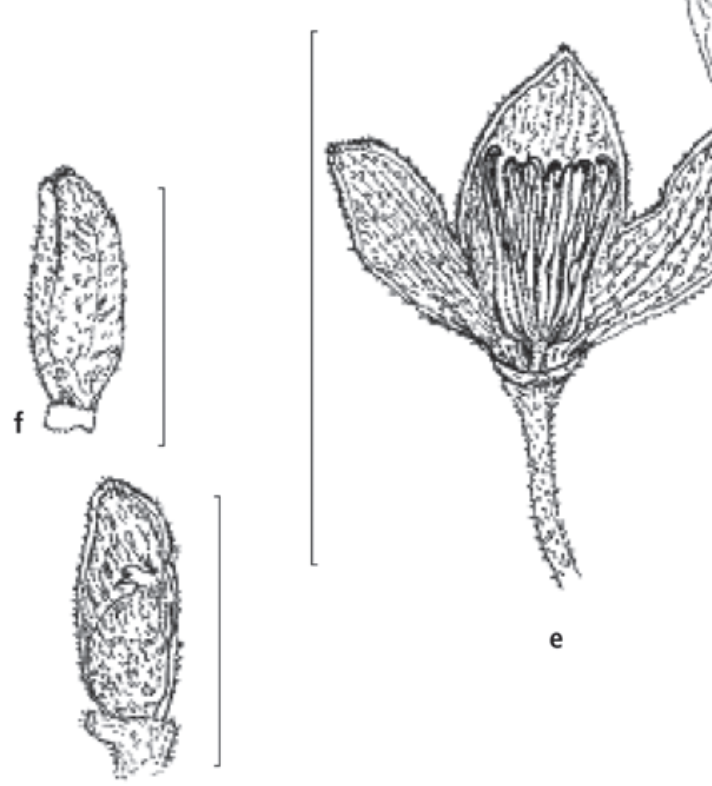

e
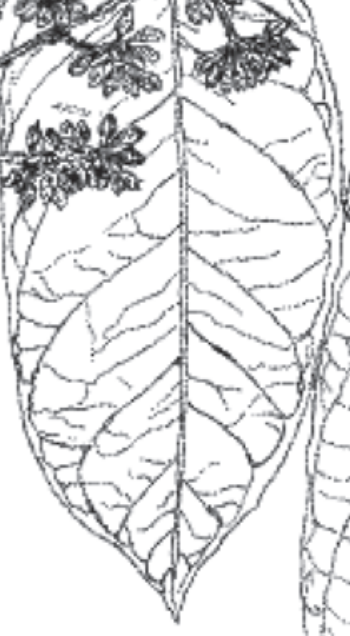
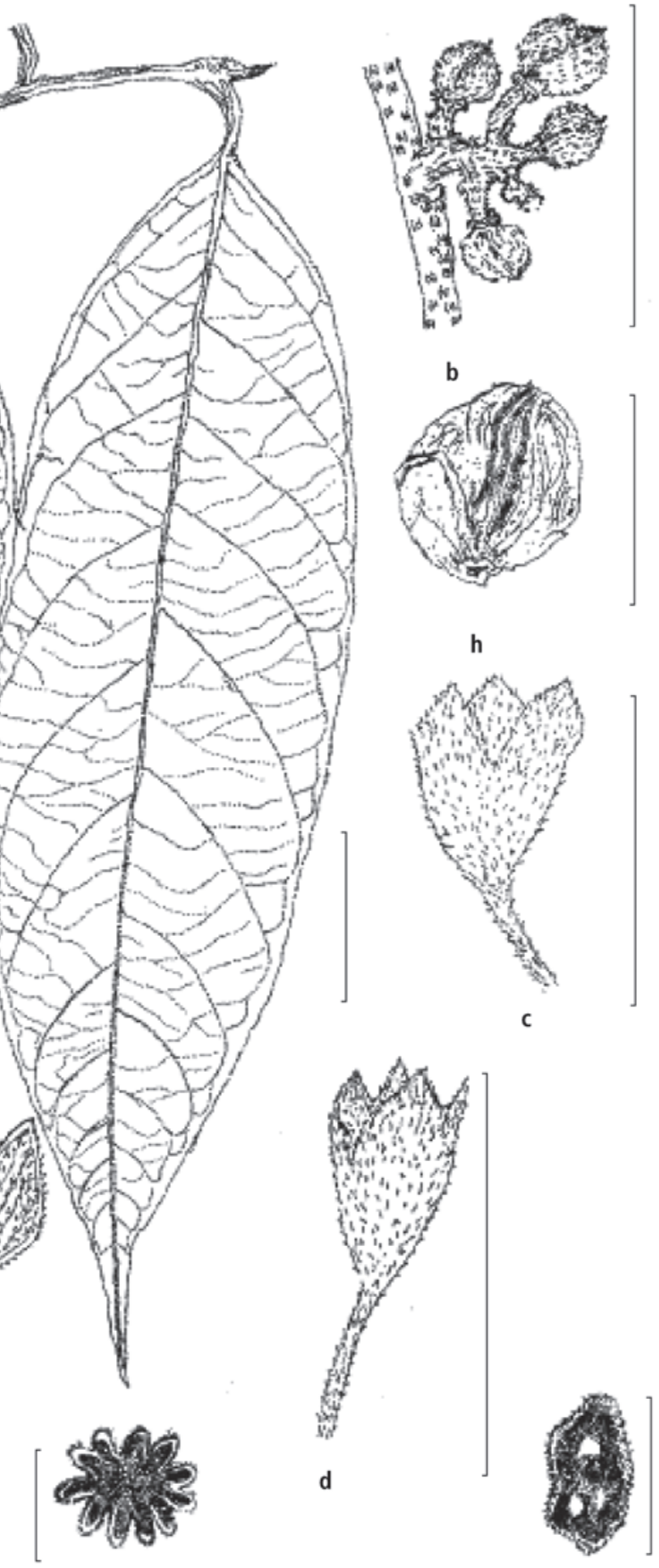

i .

j g

Fig. 1. Gymnacranthera canarica (Bedd. ex King) Warb.; a. Male flowering-twig; b. Young infructescence; c, d. Male flowers; e. L.S. of male flower with synandrium; f. Female flower bud; g. L.S. of female flower bud; h. Mature fruit; i. T.S. of synandrium; j. T.S. of ovary. [a,c-e \& i from N.C. Nair 77268 (CAL); b from C.N. Mohanan 63746 (CAL); f,g,j from C.N. Mohanan 61234 (CAL), h from P. Bhargavan 87472 (CAL)] Scale bars: $\mathrm{a}=1 \mathrm{~cm}, \mathrm{~b}=1.8 \mathrm{~cm}, \mathrm{c}-\mathrm{e}=8 \mathrm{~mm}, \mathrm{f}-\mathrm{g}=3.5 \mathrm{~mm}, \mathrm{~h}=2.5 \mathrm{~cm}, \mathrm{i}=2 \mathrm{~mm}$, $\mathrm{j}=1.5 \mathrm{~mm}$. 
Note: Gymnacranthera farquhariana (Hook.f. \& Thomson) Warb. differs from G. canarica in having incurved apical portions of free anthers and ellipsoid fruits, and is distributed in Peninsular Thailand, Malaya, Singapore, S. Sumatra and Borneo. Moreover, it does not occur in India.

Specimens examined: INDIA. Karnataka: Coorg, Pl. Indiae Or. (Terr. Canara et Contin), Prope Mercara, November, R.F. Hohenacker 541 (K, BM); South India, Beddome 1/68 (K); South Canara, R.H. Beddome s.n. (K). Kerala: Idukki district, Adimali RF, 1800 m, 28.3.1980, K. Ramamurthy 66558 (CAL); Kollam district, Travancore, Coluternypolay, 200 $\mathrm{ft}$, 24.2.1894, T.F. Bourdillon 93 (K); Thenmalai - Ariyankavu, 9.3.1980, C.E. Ridsdale 538 (K); Puliyar, Munnar - Alwaye Rd., ca. 15 km from Munnar, Cardamom hills, 28.3.1980, C.E. Ridsdale 755 (K); Palakkad district, Mukkali forest, 350 ft, 2.4.1983, N.C. Nair E P. Bhargavan 77268 (K); Thiruvananthapuram district, TBGT, Garden site, 28.3.1990, C. Anil Kumar 2550 (TBGT). Tamil Nadu, Tinnevelley hills, s.d., R.H. Beddome 6723 (BM).

\section{Myristica Gronov., nom. cons.}

Myristica Gronov., Fl. Orient.: 141. 1755, nom. cons.; Warb., Nova Acta Acad. Caes. Leop.-Carol. German. Nat. Cur. 68: 374. 1897; J. Sinclair, Gard. Bull. Singapore 16: 333. 1958 \& 28: 1. 1968; W.J. de Wilde, Blumea 40: 237. 1995; 42: 111. 1997; Fl. Males. Ser. 1. Spermat. 14: 359. 2000. Myristica sect. Myristica Blume, Rumphia 1: 180. 1836; Hook.f. \& Thomson, Fl. Ind. 1: 162. 1855, p.p. sect. Eumyristica; A. DC., Prodr. 14, 1: 189. 1856; Miq., Fl. Ned. Ind. 1(2): 53. 1858; Hook.f., Fl. Brit. India 5: 102. 1886; King, Ann. Roy. Bot. Gard. Calcutta 3: 286. 1891. Type: Myristica fragrans Houtt.

Trees, dioecious, tall. Androecium a stalked column, linear anthers fused in elongated synandrium, with or without sterile apex, androphore cylindrical. Aril lacerated nearly to base.

Distribution: Nearly 175 species, distributed from India to Southeast Asia to New Guinea, northeast Australia, also in Fiji Islands; only 5 species are distributed in India.

\section{Key to taxa}

(for male flowering and fruiting specimens)

1. Male inflorescence either paniculate or with few long-pedicelled flowers; peduncles 10-25 mm long . ...............

1. Male inflorescence on a 1-3-lobed woody tubercle of 5-15 mm long; peduncles 0-10 mm

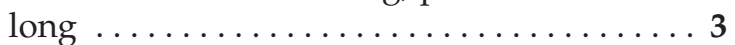

2. Leaf apex acute-acuminate; male inflorescence with 1-3 flowers; pedicels 10-15 mm long; fruits globose, 6-9 cm, early glabrescent; seeds c. $3 \mathrm{~cm}$ long (cultivated) ..... 3. M. fragrans

2. Leaf apex blunt to subacute; male inflorescence paniculate, 2-6 cm long, loosely branched; peduncles slender, 10-25 mm long; pedicels 6-9 mm long; fruits ovoid-oblong, base rounded at apex, bluntly pointed at apex, 5-6 × 4-6 cm, densely tomentose; seeds $4-4.5 \mathrm{~cm}$ long ..... ............... 5. Malabarica

3. Male pedicels slender, 6-9 $\mathrm{mm}$ long; fruits puberulous to glabrescent . 1. M. andamanica

3. Male pedicels stout, $3-8 \mathrm{~mm}$; fruits puberulous to pubescent with hairs $1-1.5 \mathrm{~mm}$ long . . . . 4

4. Leaves dendroid-hairy beneath; petioles stout, 20-50 × 3-6 mm; synandrium cylindrical with succate base; pericarp $c .4 \mathrm{~mm}$ thick ....... ............. 4. Magnifica

4. Leaves glabrous or scattered arachnoid-hairy with white-papillose or alveolar surface beneath; petioles slender, 12-30 × 2-4 mm; synandrium ellipsoid-oblong; pericarp 4-10 mm thick . . . . . . . . . . . . . . . . . . . . 5

5. Leaves $7-10 \times 3-5.5 \mathrm{~cm}$, glabrous beneath; lateral veins 7-10 pairs; fruits and seeds globose; pericarp c. $4 \mathrm{~mm}$ thick ........... .... . 2b. M. beddomei subsp. sphaerocarpa

5. Leaves $10-31 \times 5.5-12 \mathrm{~cm}$; lateral veins $10-20$ pairs; fruits and seeds ellipsoid .......6

6. Bracteoles persistent; hairs on male flowers 0.3-0.5 mm long; dry pericarp 3-4 mm thick . . . . . . 2c. M. beddomei subsp. ustulata

6. Bracteoles persistent or caducous; hairs on male flowers $0.1-0.3 \mathrm{~mm}$ long; dry pericarp 4-10 mm thick ................... . . . . . 2a. M. beddomei subsp. beddomei

1. Myristica andamanica Hook.f., Fl. Brit. India 5: 103. 1886; King, Ann. Roy. Bot. Gard. Calcutta 3: 294, t. 115. 1891; Warb., Nova Acta Acad. Caes. Leop.-Carol. German. Nat. Cur. 68: 411. 1897; Brandis, Indian Trees: 524. 1906; C.E. Parkinson, Forest Fl. Andaman Isl.: 223, f. 3. 1923; J. Sinclair, Gard. Bull. Singapore 23: 427, f. 75A-E. 1968; W.J. de Wilde, Blumea 42: 146. 1997. M. elliptica sensu Kurz, Forest Fl. Burma 2: 282. 1877, non Wall. ex Hook.f. \& Thomson, 1855. Type: INDIA, Andamans, 1884, King's Collector s.n., male fls. (lectotype, BM!) (vide de Wilde, 1997, 1.c.).

Tree, dioecious; apex of twig stout, $4.5-5 \mathrm{~mm}$ in diam., glabrous to faintly puberulous; leaves 
elliptic to broadly elliptic, $12-35 \times 4-15 \mathrm{~cm}$, acute to obtuse at base, entire at margins, acute to acuminate at apex. Inflorescences axillary or cauline, a compact subumbel on short tubercles; tubercles unbranched or bifurcate, sessile or to 1 $\mathrm{cm}$; peduncles $0.5-1.3 \mathrm{~cm}$ long, tomentose with dendroid hairs; male inflorescence 6-9-flowered; male flower pedicel slender, 6--9 $\mathrm{mm}$ long, tomentose; buds obovoid to ellipsoid, longer than broad, 3.5-4.5 × 3-4.5 mm, tomentose, valves 3 , splitting nearly $1 / 3$ rd to $2 / 3$ rds of perianth at anthesis; lobes glabrous inside, involute at anthesis; androecium 3.5-4 mm long; synandrium cylindrical, 2-2.5 mm long; anthers 6-10, sterile apiculus blunt, to $0.3 \mathrm{~mm}$ long; androphore slender, 1-1.5 mm long, glabrous to puberulous; female flowers sessile, pubescent; stigma sessile, 2-lobed; fruits ovoid to ovoid-oblong, 4.5-6.5 $\times$ 2.5-3.5 cm, obtuse at apex, brown-puberulous (hairs c. $0.1 \mathrm{~mm}$ long) to glabrous; pericarp 5-10 $\mathrm{mm}$ thick; aril lacerated nearly to base, red; seeds ellipsoid.

Flowering: June-July; Fruiting: February-March; September.

Habitat: Subtropical evergreen inland forests, sometimes near shores.

Distribution: India (Andaman and Nicobar Islands), also grown in division number 25 of AJC Bose Indian Botanic Garden, Howrah, West Bengal (Chowdhery \& Pandey, 2007). Endemic.

IUCN Threat Status: Vulnerable B1+2c ver. 2.3 (IUCN, 2017-1).

Specimens examined: INDIA, Andaman and Nicobar Islands: Andamans, G. King 6/85 (1884) (K); Andamans, 1884, G. King's Collector s.n. (CAL); South Andaman, s.d., S. Kurz 9 (K); S. Andamans, s.d., S. Kurz s.n. (CAL, K); South Andaman, s.d., S. Kurz 265 (CAL); South Andaman, Hutbaypur, 6.9.1890, G. King's Collector s.n. (CAL, K); South Andaman, North Bay, near shore, 12.9.1891, G. King's Collector s.n. (CAL); Corbycis Core (North), 13.2.1892, G. King's Collector s.n. (CAL, BM); Anikhet-Hill Jungle, 5.3.1893, G. King's Collector s.n. (CAL, K); Mount Harriet, 2.3.1895, S. Kurz s.n. (CAL); Long Islands, May 1915, C.E. Parkinson 636 (= 669A) (CAL); Middle Andaman, Claudine Range, c. 213 m, 6.4.1916, C.E. Parkinson 1170 (CAL).

2. Myristica beddomei King, Ann. Roy. Bot. Gard. Calcutta 3: 291, t. 118, f. 1-8. 1891; W.J. de Wilde, Blumea 42: 149. 1997.

Note: Three subspecies have been recognized in Myristica beddomei, viz., subsp. beddomei, subsp. ustulata and subsp. sphaerocarpa and all are distributed in India (de Wilde, 1997, 1.c.). Myristica beddomei subsp. beddomei and subsp. ustulata were inadvertently identified as $M$. dactyloides Gaertn. in several herbarium specimens and in regional floras. Myristica dactyloides differs from $M$. beddomei in having broadly lanceolate to broadly elliptic leaves with obtuse base, brownish with indistinct veins beneth, male flowers with caducous bracteole and distinctly ellipsoid male flower buds with pointed apex, and M. dactyloides is endemic to Sri Lanka.

2.a. subsp. beddomei: King, Ann. Roy. Bot. Gard. Calcutta 3: 291, t. 118, f. 1-8. 1891; Talbot, Syst. List Trees Bombay: 165. 1894 \& Forest Fl. Bombay 2: 380. 1911; Warb., Nova Acta Acad. Caes. Leop.Carol. German. Nat. Cur. 68: 504. 1897; Gamble, Man. Ind. Timb.: 556. 1902; T. Cooke, Fl. Bombay: 530. 1906; Gamble, Fl. Madras: 1214. 1925; T.S. Nayar et al., Fl. Pl. Western Ghats 1: 663. 2014 p.p. excl. syn. Myristica laurifolia Hook.f. \& Thomson. Lectotype (designated here): King t. 118. [vide King, 1891, 1.c.].

Myristica laurifolia auct. non Hook.f. \& Thomson, 1855: Bedd., Fl. Sylv. S. India: t. 267. 1872, p.p. quoad fig. fruit; Talbot, Syst. List Trees Bombay: 165. 1894.

Myristica contorta Warb., Nova Acta Acad. Caes. Leop.-Carol. German. Nat. Cur. 68: 507, t. 16, f. 1-3. 1897. Lectotype,: INDIA, Karnataka, North Canara, Devimane ghat, 20.11.1882, Talbot 225 (male fl.) (K!) (vide de Wilde, 1997, 1.c.).

Myristica laurifolia Hook.f. \& Thomson var. lanceolata Hook.f., Fl. Brit. India 5: 103. 1886. Holotype: INDIA, Karnataka to Tamil Nadu, Canara to Cape Coumarin, up to $1525 \mathrm{~m}$, March 1872, Beddome 263 (K!).

Myristica dactyloides sensu J. Sinclair, Gard. Bull. Singapore 23: 445, f. 78. 1968, p.p., non Gaertn, 1788, excl. specimens from Sri Lanka and India possibly mixed with subsp. ustulata.

Vernacular Names: Kannada: Jajikai, Ramnadike; Malayalam: Patthapanu; Marathi: Jayaphal; Tamil: Katjathikai.

Tree, dioecious, occasionally with knee roots; apex of twigs stout, $2-4 \mathrm{~mm}$ in diam., striate, glabrous to faintly puberulous; leaves broadly elliptic to oblanceolate, $10-30 \times 3-5.5 \mathrm{~cm}$, rounded to cuneate at base, entire at margins, acute to acuminate at apex, glabrous beneath; lateral veins 12-20 pairs; petioles $10-30 \times 2-4 \mathrm{~mm}$. Inflorescence axillary or cauline, compact subpaniculate, up to 6 flowers on tubercles; tubercles 5-15 $\mathrm{mm}$ long, browntomentose (hairs $0.1-0.4 \mathrm{~mm}$ long); peduncles 
3-10 cm long; flowers up to 30 in different stages of development; male flower pedicel stout, 2.5-5 $\mathrm{mm}$; hairs 0.1-0.4 mm long; flower buds ovoid to ellipsoid, longer than broad, 4-6 $\times 2.5-3 \mathrm{~mm}$, tomentose, 3 valves, splitting nearly to $1 / 3$ rd to $2 / 3$ rds of perianth at anthesis; lobes glabrous inside, involute at anthesis; androecium slender, 3.5-4.5 $\mathrm{mm}$ long; synandrium ellipsoid-oblong, 1.8-2.5 $\times$ 0.8-1.3 mm; anthers 6-10 with blunt sterile apex of $0.1-0.5 \mathrm{~mm}$ long; androphores cylindrical, 1.8-2 $\times 0.7-1 \mathrm{~mm}$, glabrous to stellate-puberulous (hairs 0.1-0.2 mm long); pedicels in female flowers stout; flower buds ellipsoid, tomentose; stigma sessile, 2-lobed; fruits broadly subglobose, $4.5-5 \times(2.5-) 3-4$ $\mathrm{cm}$, brown-pubescent (hairs $0.1-0.2 \mathrm{~mm}$ long); (dry) pericarp 4-10 mm thick; fruit stalks 5-10 mm long; aril lacerated nearly to base, red; seeds ellipsoid.

Flowering: January-April; October-November; Fruiting: May-July.

Habitat: In subtropical evergreen forests, along the sholas and ghats, occasionally in Myristica swamps.

Distribution: India (Karnataka, Kerala, Maharashtra and Tamil Nadu). Endemic.

Uses: Reddish grey wood is moderately hard and useful for boxes of tea, match and for splints.

Specimens examined: INDIA, Penninsula Indiae Orientalis, Herb. R. Wight (CAL, K); Peninsular Ind. Orientalis, Herb. Wight Propr. 2814 (E). Karnataka: Mysore, 1837, ex herb. H.F.C. Cleghorn s.n. (E); Hassan district, Bisle ghat, Parorama view point, 17.1.1969, C.J. Saldanha 14203 (E); Biccode to Arehalli Rd., 17.9.1969, C.J. Saldanha 15089 (E); Kodagu district, Coorg, Bhimangudi, Sampaje Ghat Forest, 2500 m, 23.10.1963, A.S. Rao 95017 (CAL); Shimoga district, Agumbe, Someswar ghat, 6.2.1961, R.S. Raghavan 69364 (CAL). Kerala: Idukki district, Santhanpara, $5000 \mathrm{ft}$, Dec. 1909, A. Meebold (741) 13134 (CAL); Kollam district, Achancoil - Thorai, 400 m, 26.5.1979, C.N. Mohanan 63039 (CAL); Palakkad district, Silent valley, 25.6.1876, C.E. Ridsdale 284 (K); Mukkali, Mandampatty forest - Mukkali, 525 m, 6.4.1978, N.C. Nair 56620 (CAL); Silent Valley, eastern slopes, $950 \mathrm{~m}, 17.1 .1980, P$. Bhargavan 65526 (CAL); Thiruvananthapuram district, Vendamettu, 5000 ft, Dec. 1909, A. Meebold (971) 12964 (CAL); Palode, Bonaccord, $700 \mathrm{~m}$, 3.4.1990, N. Mohanan 8161 (TBGT); Ponmudi, 6.3.1880, C.E. Ridsdale 476 (K); Wayanad district, s.loc., 1857, Drew s.n. (E). Maharashtra: Bombay, N.A. Dalzell s.n. (K); Ellora nulleys, $3000 \mathrm{ft}$, 26.11.1888, M.A. Lawson s.n. (K). Tamil Nadu: Coimbatore district, Anamalais, specimen A, R.H. Beddome s.n. (BM); Anamalais, Iyerpadi, 25.10.1901,
C.A. Barber 3869 (K); Udumanparai, 22.11.1901, C.A. Barbar 4108 (K); 16.5.1903, C.A. Barber 5911 (CAL); Karian Shola (Top slip), near Coimbatore, c. $1000 \mathrm{~m}, 2.7 .1976$, A.J.G.H. Kostermans 26240 (K); Dindigul district, Lower Pulneys, Tandigudi, 6.6.1901, Bourne 2093 (CAL, K); Nilgiris district, Sispara Ghat, 5000 ft, Nov. 1883, J.S. Gamble 13415 (K); Gudalur ghat, 4000 ft, Oct. 1886, J.S. Gamble 18294 (BM); Tirunelveli district, Tinnevelly ghat, upper specimen, R.H. Beddome s.n. (BM); Kannikatti Jemin, May 1901, C.A. Barber 2942 (K).

2.b. subsp. sphaerocarpa W.J. de Wilde, Blumea 42: 152. 1997.

Type: INDIA, S. India, Western Ghats, Tamil Nadu, Tirunelveli district, eastern slopes of Ghats, Walaiyar cardamom estate, $1100 \mathrm{~m}, 11.7 .1976$, Kostermans 26276(a) (holotype, L, n.v.; isotype, K!).

This subspecies differs from the typical subspecies in its leaf size $(7-10 \times 3-5.5 \mathrm{~cm})$, number of lateral veins (7-10 pairs), globose fruits and seeds, and $c$. $4 \mathrm{~mm}$ thick pericarp.

Fruiting: January-July.

Habitat: Subtropical evergreen forests, along the sholas and ghats, occasionally in Myristica swamps.

Distribution: India (Karnataka, Kerala and Tamil Nadu). Endemic.

IUCN Threat Status: Endangered B1+2c ver. 2.3 (IUCN, 2017-1).

Uses: Wood reddish grey, perishable, suitable for match boxes and splints (CSIR, 1956).

Specimens examined: INDIA, Karnataka: Hassan district, Malgod, 23.1.1970, C.J. Saldanha 16136 (K, E). Kerala: Palakkad district, Panthenthode, 850 m, 3.4.1983, N.C. Nair 77291 (CAL, K). Tamil Nadu: Namakkal district, Kolli hills, Ariyur (Periasamikoil) Shola, 1300 m, 20.4.1979, K.M. Matthew 22938 (K); Tirunelveli district, Tinnevelley ghat, s.d., s.coll., s.n. (BM).

2.c. subsp. ustulata W.J. de Wilde, Blumea 42: 152. 1997.

Type: INDIA, S. India, E. Madras, Anamalais, Karian Shola, 500 m, 29.10.1974, A.J.G.H. Kostermans 25825 (holotype, L, n.v.; isotypes, K!, BM!).

It differs from the typical subspecies in having 3-4 $\mathrm{mm}$ thick dry pericarp; male flowers with $0.3-0.5$ $\mathrm{mm}$ long blackish brown hairs and persistent bracteole.

Flowering \& Fruiting: August-November. 
Habitat: Subtropical evergreen forests, along the sholas and ghats, occasionally in Myristica swamps.

Distribution: India (Kerala and Tamil Nadu). Endemic.

IUCN Threat status: Endangered B1+2c ver. 2.3 (IUCN, 2017-1).

Note: A mixture of M. beddomei subsp. ustulata and M. beddomei subsp. beddomei was inadvertendly treated as M. dactyloides Gaertn. by Gandhi (1978) in Flora of Hassan District, Karnataka. In fact, $M$. dactyloides is confined to Sri Lanka and does not occur in India.

Specimens examined: INDIA, Kerala: Herb. R. Wight s.n. Prop. Presented 1871 (K); Kollam district, Colatoorpolay, $2500 \mathrm{ft}, 28.11 .1893$, M.A. Lawson 94 (K, CAL); Wyanad district, SE Wynaad, above Nilgris, 188(4), M.A. Lawson 1 (K). Tamil Nadu: Coimbatore district, Annamallays, R.H. Beddome s.n. (specimen B, lower one) (BM); Dindigul, Kodaikanal taluk, Thadiankudisai, 1050 m, 3.8.1988, K.M. Matthew 53298 (K); Nilgiris district, Sispara Ghat, 5000 ft, June 1884, J.S. Gamble 14466 (K); Gudalur Ghat, 5000 ft, Oct. 1884, J.S. Gamble 14912 (K); Gudalur Ghat, 4000 ft, Oct. 1886, J.S. Gamble s.n. (K); Tirunelveli district, Kannikatti Jemin, May 1901, C.A. Barber 2941 (K).

3. Myristica fragrans Houtt., Nat. Hist. 2(3): 333. 1774; Blume, Rumphia 1: 180, t. 55. 1835; A. DC., Prodr. 14(1): 189. 1856; Miq., Fl. Ned. Ind. 1(2): 53. 1858; Hook.f., Fl. Brit. India 5: 102. 1886; King, Ann. Roy. Bot. Gard. Calcutta 3: 287, t. 108. 1891; Warb., Muskatnuss: 271. 1897 \& Nova Acta Acad. Caes. Leop.-Carol. German. Nat. Cur. 68: 458. 1897; Gamble, Mat. Fl. Malay Penins. 5(23): 233. 1912; Burkill, Gard. Bull. Straits Settlem. 2: 158. 1918; A.C. Joshi, J. Indian Bot. Soc. 25: 139. 1946; Ridl., Fl. Malay Penins. 3: 63. 1924; J. Sinclair, Gard. Bull. Singapore 16: 361, f. 29, t. VIIB. 1958 \& 23: 225. 1968; W.J. de Wilde, Blumea 35: 244. 1990 \& Fl. Males. Ser. 1, Spermat. 14: 470. 2000; B. Li \& T.K. Wilson, Fl. China 7: 99. 2009; T.S. Nayar et al., Fl. Pl. Western Ghats 1: 664. 2014. Lectotype: J.W. Weinmann, Taalrijk Register 7, pl. 760a. 1748 (de Wilde, 2000, l.c.).

Myristica officinalis L.f., Suppl. Pl.: 265. 1781; Gaertn., Fruct. Sem. Pl. 1: 194, t. 41, f. 1. 1788, p.p. excl. nux myristica mas. Rumphia; Hook., Bot. Mag. (Kew Mag.) 54: f. 2756 \& 2757. 1827. Type: (Indonesia, Hospitatur in Insula Banda, Nordberg Herb. Linn. 1204.1 BM!).

Myristica philippinensis Gand., Bull. Soc. Bot. France 66: 226. 1919. Type: SINGAPORE, Cuming
2418 (holotype, LY, n.v.; isotypes, BM!, CAL!, K!). Fig. 2a-e

Vernacular Names: Hindi, Bengali, Marathi, Gujarati: Jaiphal - fruit kernel; Japatri,Jotri, Jayapatri, Joitri - aril; Telugu, Tamil, Kannada, Malayalam: Jajikai, Jadikai - fruit kernel; Jadipattiri, Japatri - aril (CSIR, 1956).

Tree, dioecious; apex of twigs slender, striate, glabrous; leaves elliptic to oblong, 6-15 × 3.5-6.5 $\mathrm{mm}$, attenuate at base, entire at margins, acuteacuminate at apex, glabrous beneath; lateral veins $5-12$ pairs; petioles $5-12 \times 0.7-1.5 \mathrm{~mm}$; male inflorescence axillary or supra-axillary, pedunculate; peduncles slender, 5-20 mm long, one apical flower and 1 or 2 flowers apical opposite short-shoots; pedicels slender, 10-15 mm long; buds ellipsoid, longer than broad, $7-8 \times 4-5$ $\mathrm{mm}$, valves 3 ; splitting nearly to $1 / 3 \mathrm{rd}$ to $2 / 3 \mathrm{rds}$ of perianth at anthesis; lobes glabrous, involute at anthesis; androecium slender, ellipsoidal, 5-6 mm long; synandrium c. $3 \times 1-1.5 \mathrm{~mm}$; stamens 7-12, sterile apex subacute, c. $0.5 \mathrm{~mm}$ long; androphore 2-2.5 × c. $1 \mathrm{~mm}$, puberulous to glabrous; hairs c. 0.1 $\mathrm{mm}$ or less; female flower buds ovoid, glabrous; stigma subsessile, bilobed; fruit stalks $10-15 \mathrm{~mm}$ long; fruits (sub)globose, 6-9 cm in diam., early glabrescent; pericarp 10-15 mm thick (dry); aril lacerated nearly to base, red; seeds ellipsoid or globose, c. $3 \mathrm{~cm}$ long.

Flowering \& Fruiting: Throughout the year.

Habitat: Cultivated in subtropical evergreen forests, along the ghats and botanical gardens.

Distribution: Native to East Indonesia. Cultivated in the tropics throughout the world, viz., India, Sri Lanka, China, S. Vietnam, Indonesia, Peninsular Malaysia, Philippines, Africa, West Indies and South America. In India, it is cultivated in West Bengal, Tamil Nadu, Kerala and Andaman and Nicobar Islands.

IUCN Threat Status: Data Deficient ver. 2.3 (IUCN, 2017-1).

Uses: Nutmeg with mace of commerce is used as spice and condiments and in herbal medicines. The seeds contain essential and fixed oils. Essential oils contain a pale yellow liquid containing eugenol, d-camphene, geraniol, free myristic acid and the narcotic myristicin. It is used as carminative and flavouring agent. The fixed oil of nutmeg butter is highly aromatic, used as mild stimulant for external application and in the manufacturing of perfumes (Sinclair, 1958).

Specimens examined: East India, W. Roxburgh, Herb. Forsynth, Purchased 1835 (K); Peninsula Indiae 

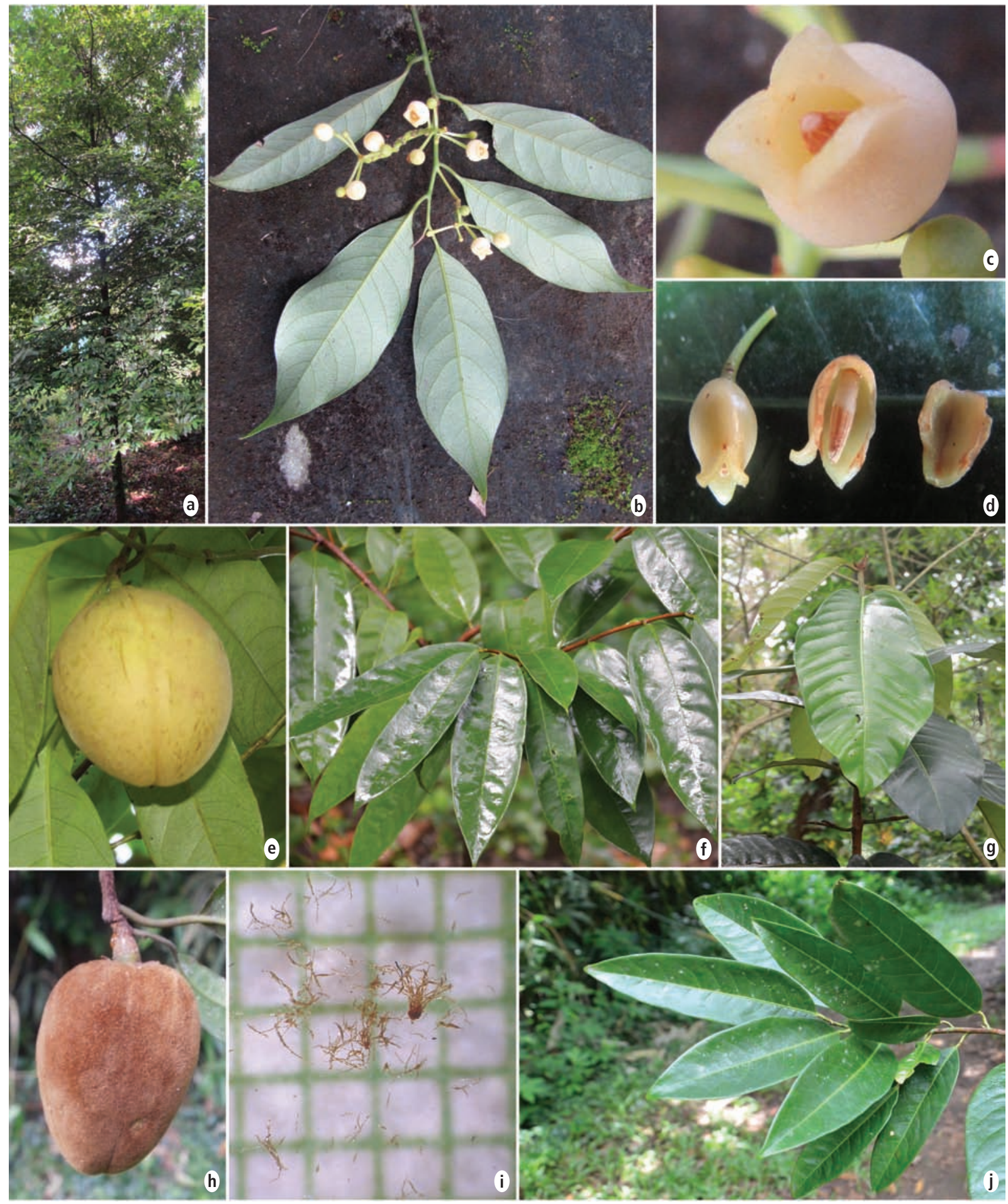

Fig. 2. A-E. Myristica fragrans Houtt.: a. Habit; b. Male flowering twig; c \& d. Male flower; e. Fruit; f. Gymnacranthera canarica (Bedd. ex King) Warb. - Young plant; g. M. magnifica Bedd. - Young shoot; h-j. M. malabarica Lam. - h Fruit; i. Hairs on fruit; j. Leaves. (Scale bars $=1 \mathrm{~mm}$ as shown in c, d \& i) 
Orientalis, No. 669 Ex herb. Hort. Bot. Calcuttensis, Herb. R. Wight No. 2488 (K, E); Peninsular Ind. Orientalis, Wight Proper 2814 (E). Kerala, Cochin, Bolghat, 9/84, N. Gam, Cult, J.S. Gamble s.n. (K); Thiruvananthapuram district, TBGT, cultivated, 10.10.2002, Mathew Dan 47714 (TBGT); Brimose estate, 9.11.1995, P. Deepti Das 26290 (TBGT); Palki, Chenara, 7.12.1985, Binoy P.C. (20739) 185 (TBGT); University of Calicut Botanic Garden, 26.1.1994, M. Chorley \& V. Bharatan 52 (BM). Tamil Nadu, Peninsular Ind. Orientalis, Tinnevelley, Courtallum, s.d., Herb. R. Wight Proper 722 (E); Tinnevelley, Courtallam, Herb. Hookerianum, s.d., Wall. Numer. List No. 6785A (K); Courtallam, 1822, HBC(?)Wall. Numer. List No. 6785; Burliyar, 2000 $\mathrm{ft}$, Jan. 1883, J.S. Gamble 11558 (K); Courtallum in gardens, 1885, R.H. Beddome 6732 (BM).

4. Myristica magnifica Bedd., Fl. Sylv. S. India 2: 268, t. 268. 1872; Gamble, Man. Ind. Timb.: 314. 1881; Hook.f., Fl. Brit. India 5: 104. 1886; King, Ann. Roy. Bot. Gard. Calcutta 3: 291, t. 119. 1891; Warb., Nova Acta Acad. Caes. Leop.-Carol. German. Nat. Cur. 68: 424. 1897; Talbot, Syst. List Trees Bombay ed. 2: 280. 1902 \& Forest Fl. Bombay 2: 381. 1911; T. Cooke, Fl. Bombay: 531. 1906; Brandis, Indian Trees: 524. 1906; Gamble, Fl. Madras: 1214. 1925; W.J. de Wilde, Blumea 42: 174. 1997.

Myristica fatua Houtt. var. magnifica (Bedd.) J. Sinclair, Gard. Bull. Singapore 23: 282, f. 33. 1968; T.S. Nayar et al., Fl. Pl. Western Ghats 1: 664. 2014. Syntypes: INDIA, Kerala: Kollam, Ghats around Minmoote, near Colatoorpolay, Beddome s.n. (K!); Kerala \& Tamil Nadu, South Travancore, s.d., Beddome s.n.; s.loc., March 1872 (3/72), Beddome 242 (K!). Tamil Nadu, Courtallam Hills, 1873, Beddome s.n. (K!); Tinnevelly ghats, 1885, Beddome 6725, 6726 (BM!).

Fig. $2 \mathrm{~g}$

Vernacular Name: Malayalam: Kattha panu.

Tree, dioecious with knee roots; twigs stout, apex striate, brown-tomentose to glabrous; hairs $0.5-1$ $\mathrm{mm}$ long; leaves elliptic to oblong-lanceolate, $20-60 \times 6-20 \mathrm{~cm}$, rounded at base, entire at margins, bluntly acute to acute-acuminate at apex, puberulous-brown beneath; hairs dendroid, 0.3-1 mm long, late caducous; lateral veins $15-27$ pairs; petioles $2-5 \times 0.25-0.6 \mathrm{~cm}$, puberulous to glabrous; inflorescence a tubercle, brown-pubescent; hairs $0.5-1.5 \mathrm{~mm}$ long; subsessile or pedunculate to $5 \mathrm{~mm}$ long; tubercle unbranched to branched twice; subumbels 3-20-flowered; flowers in different stages of development, tomentose, brown; hairs c. $1 \mathrm{~mm}$ long; male flower pedicels stout, 3-8 mm; buds ovoid-ellipsoid, longer than broad, 5-8 $\times 4-5 \mathrm{~mm}$, valves 3 ; splitting nearly to $1 / 3$ rd to $2 / 3$ rds of perianth at anthesis; lobes glabrous inside, involute at anthesis; androecium cylindrical, \pm saccate at base, $4-4.5 \times 0.8-1 \mathrm{~mm}$, synandrium cylindrical; sterile apex $0-0.1 \mathrm{~mm}$ long; androphore cylindrical, $1-1.5 \times$ c. $0.7 \mathrm{~mm}$, furrowed, glabrous at apex, densely tomentose at base, hairs c. $0.5 \mathrm{~mm}$ long; female flower buds ovoid, tomentose, brown; hairs c. $1 \mathrm{~mm}$ long; stigma sessile, 2-lobed; fruit stalks $2-5 \mathrm{~mm}$ long; fruits ovoid-oblong, $4.5-5.5 \times 2.5-3.5 \mathrm{~cm}$, broadly rounded at base, blunt at apex, densely brownpubescent; hairs 1-1.5 mm long; pericarp c. $4 \mathrm{~mm}$ thick (dry); aril lacerated nearly to base, red; seeds ovoid-oblong, c. $4.5 \mathrm{~cm}$ long.

Flowering: May-November; Fruiting: OctoberMarch.

Habitat: (Sub)tropical evergreen forests, common in Myristica swamps.

Distribution: Kerala and Maharashtra. Endemic.

IUCN Threat Status: Endangered B1+2c ver. 2.3 (IUCN, 2017-1).

Specimens examined: INDIA, Karnataka: N. Canara, Melamane Ghat, 1000 ft, Oct 1919, Ambo 7191 (Herb. L.J. Sedgwick \& T.R.D. Bell) (CAL). Kerala: Thenmalai - Ariyankavu, 9.3.1880, C.E. Ridsdale 539 (K); Travancore, 26.1.1890, T.F. Bourdillon 730 (1639) (CAL, TBGT); Colatoorpolay, 25.2.1893, M.A. Lawson s.n. (CAL); Madras, Travancore, M.A. Lawson 90(b) (CAL); Tranvancore, Kollam district, Kulathupuzha (Colatoorpolay), 26.11.1893, M.A. Lawson s.n. (CAL, K); Travancore, M.A. Lawson s.n. (CAL); Travancore, s.d., M.A. Lawson s.n. (CAL); Travancore, forests near Colaturpolay, 26.1.1896, T.F. Bourdillon 731 (CAL, K); Forests near Colaturpolay, 26.1.1896, T.F. Bourdillon 200/2 (K); Ponmudi, 1000 m, 11.6.1976, A.J.G.H. Kostermans 26091 (K); Trivandrum, Sasthanada, 8.2.2008, C.G. Vishnu 63001 (TBGT). Tamil Nadu, Courtallum Hills, 1873, R.H. Beddome s.n. (K).

5. Myristica malabarica Lam., Mém. Acad. Sci. (Paris): 162. 1791; Encycl. 4: 388. 1797 \& Encycl. 2: 5. 1800, p.p. excl. t. 833, f. 2a-b; Hook.f. \& Thomson, Fl. Ind. 1: 163. 1855; A. DC., Prodr. 14(1): 194. 1856; Drury, Useful Pl. India: 305. 1858; Dalzell \& A. Gibson, Bombay Fl.: 4. 1861; Drury, Handb. Ind. Fl. 3: 78. 1869; Bedd., Fl. Sylv. S. India 2: t. 269. 1872; Gamble, Man. Ind. Timb. ed. 2: 314. 1881 \&: 555. 1902; Hook.f., Fl. Brit. India 5: 103. 1886; King, Ann. Roy. Bot. Gard. Calcutta 3: 288, t. 109. 1891; Talbot, Syst. List Trees Bombay: 165. 1894; Warb., Nova Acta Acad. Caes. Leop.-Carol. German. Nat. Cur. 68: 403, t. 12, f. 1-8. 1897, p.p. excl. M. heyneana; T. Cooke, Fl. Bombay: 530. 1906; Brandis, 
Indian Trees: 524. 1906; Talbot, Forest Fl. Bombay 2: 378, f. 459. 1911; Gamble, Fl. Madras: 1213. 1925; J. Sinclair, Gard. Bull. Singapore 23: 168, f. 9. 1968; W.J. de Wilde, Blumea 42: 175. 1997; T.S. Nayar et al., Fl. Pl. Western Ghats 1: 664. 2014. Lectotype (designated here): Rheede, Hort. Malab. 4: 9, 10, t. 5.1683.

Myristica dactyloides Wall., Numer. List No. 6786. 1832, nom. nud., non Gaertn., 1788.

Voucher Specimen: INDIA, Tamil Nadu, Fl. Madras, (U keya) E.I.C., Wallich. Numer. List No. 6786, male \& female (BM!; K-W!).

Myristica notha Wall., Numer. List. No. 6787. 1832, nom. nud. Voucher Specimen: Kerala, Wild nutmeg from Aleppe, Hb. Madr., Wall. Cat. 6787 (K-W!).

Myristica tomentosa sensu J. Graham, Cat. Pl. Bombay: 175. 1839, p.p., non Thunb, 1782.

Fig. $2 \mathrm{~h}-\mathrm{j}$

Vernacular Names: Hindi: Jangli-jaiphal, Kaiphal, Ranaiphal, Rampatri - only mace; Kannnada: Pindikai - only seeds; Malayalam: Panam-palka, Ponnampanau; Tamil: Patthiri.

Tree, dioecious with knee roots; apex of twig slender, striate, puberulous to glabrous; leaves elliptic, $5-16 \times 2-5 \mathrm{~cm}$, cuneate at base, entire at margins, subacute at apex, glabrous; lateral veins 6-9 pairs; petioles slender, 5-15 × 1-2 mm; inflorescence paniculate, glabrous to pubescent, grey-brown, hairs $0.1-0.2 \mathrm{~mm}$, persistent, loosely branched; peduncles slender, $10-25 \times 1-1.5 \mathrm{~mm}$, laterals $2-10$ $\mathrm{mm}$, central branch up to $15 \mathrm{~mm}$ long; flowers 5-8, in different stages of development, clustered in loose umbels; male flower pedicel slender, 6-10 mm long; buds ovoid, longer than broad, 4-5 $\times$ 3-3.5 mm; valves 3 ; splitting nearly to $1 / 3 \mathrm{rd}$ to $2 / 3$ rds of perianth at anthesis; lobesglabrous inside, involute at anthesis; androecium narrowly conical, 3-4 × 1-1.2 mm; synandrium conical, 2.5-3 × 1-1.2 $\mathrm{mm}$, sterile apex blunt, c. $0.2 \mathrm{~mm}$ long, glabrous; anthers 6 or 7; androphore broadly cylindrical, $c$. $1 \times 1.2 \mathrm{~mm}$, densely pubescent, brown; hairs $c .0 .5$ $\mathrm{mm}$ long; fruit stalks 2-3 mm long; female flower buds ovoid, blunt at apex; glabrous to pubescent; stigma sessile, bilobed; fruits ovoid-oblong, 5-6 $\times$ 4-6 cm, rounded at base, bluntly pointed at apex (apiculate), brown-tomentose; hairs 1-1.5 mm long; pericarp c. $4 \mathrm{~mm}$ thick (dry); aril lacerated nearly to base, red; seeds ovoid-oblong, 4-4.5 cm long.

Flowering: November-May; Fruiting: July-August.

Habitat: Subtropical evergreen forests, common in Myristica swamps
Distribution: Peninsular India (Karnataka, Kerala Maharashtra and Tamil Nadu). Endemic.

IUCN Threat Status: Vulnerable B1+2c ver. 2.3 (IUCN, 2017-1).

Use: Used as adulterant of nutmeg.

Note: Regarding the specimens of Myristica malabarica, Lamarck (1791) stated that "Panampalca. Rheede mal. 4, p. 9 t. V; Nux indica oblonga intresecus similis naci moschatae. J.B. hist. I, p. 399; Nux myristica major spuria malabarica. Raj hist. 1524; Nux myristica spuria Pluk alm. 265". [Oblong Indian nut with similar internal strong smell, Greater fake Malabar Mystica nut etc.] Therefore, among these illustrations the above mentioned tabula (l.c.) is lectotypified.

Specimens examined: INDIA, Karnataka: Coorg, Madikeri, Prope Mercara, Pl. Indiae Or. (Terr. Canara), 1851, R.F. Hohenacker (515) (K, E); Mercara, Feb. 1851, R.F. Hohenacker 289a (BM); Madas Presidency, R.H. Beddome/73 (K); S. Canara, R.H. Beddome 1/68 (K); R.H. Beddome 8/79 (K); Malabar, S. Canara, R.H. Beddome 6714 (BM); North Canara, Wuddee Ghat, M. malabarica of Beddome's plates, 15.2.1883, W.A.Talbot 302 (CAL); North Canara, Wuddee Ghat, 15.2.1883, W.A. Talbot 301 (Wall. Cat. 6786) (CAL); North Kanara, Wuddee, 12.11.1883, W.A. Talbot 302 (E); N. Canara, Bombay, Mulasnani, 21.1.1896, W.A. Talbot 3720 (K). Kerala, Kollam district, Peninsula Ind. Orientalis, Quilon, December 1835, R. Wight Proper 870 (E); Peninsula Ind. Orientalis, Imleu, June 1836, $R$. Wight Proper 870 (?) (E); Colaterpolay, 26.11.1893, J. Larsen 91 (CAL); Travancore, Colaturpolay, 26.2.1894, T.F. Bourdillon 118 (CAL, K); Travancore State, Tenmalai, 400 ft, 23.3.1915, C.C. Calder 1431 (CAL); Thenmalai - Ariyankavu, 9.3.1980, C.E. Ridsdale $547(\mathrm{~K})$; Palakkad district, Vattapara to Inchikuzhi, 450 m, 29.5.1979, E. Vajravelu 62877 (CAL); Thiruvananthapuram district, TBGRI, Garden site, 10.5.1984, N. Mohanan 134 (TBGT); Athirumala, 1000 m, 12.10.1987, M. Mohanan 4247 (TBGT); Palode, riverside, 4.2.1992, A. Nazarudeen 13812 (TBGT).

\section{Doubtful Species}

Apart from that three herbarium specimens of Myristica grandiflora Wall., nom. nud. [annonated on herbarium sheets but never featured in Wallich's Numerical List], a synonym of M. philippensis Lam., vide W. Griffith, Herb. East India Co. no. 4353, BGC. (K) and N. Wallich 6800b (K, BM) revealed that Myristica philippensis was cultivated in Indian Botanic Garden, Calcutta, West Bengal. However, 
later it was not found there (Chowdhury \& Pandey, 2007) or never reported anywhere else from India. It is cultivated in Mauritius, Madagascar, Martinique and Malesia.

\section{Acknowledgements}

The authors are thankful to Director General, Council of Scientific and Industrial Research, Director, CSIR-North East Institute of Science and Technology and Director, Botanical Survey of India for logistics for the work, and to the SERB, Department of Science and Technology, Govt. of India for Grants-in-Aid (Project file no. SR/ FT/LS-39/2011) and Ministry of Environment Forest and Climate Change, Govt. of India, to the Keepers/In-charges of the herbaria mentioned in the text for providing working facilities during their visits. Authors are grateful to W.J.J.O. de Wilde and Brigitta E.E. Duyfjes, Rikjherbarium, Leiden, for correcting the manuscript to a great extent. The first author is also grateful to $\mathrm{Dr}$ M Sanjappa, former Director, BSI for initial assignment and encouragement for the work during 1999-2000.

\section{Literature Cited}

Banik, D. \& P.P. Bora 2016a. Taxonomic notes on Indian Horsfieldia and Endocomia (Myristicaceae). Jap. J. Bot. 91(3): 160-178.

Banik, D. \& P.P. Bora, 2016b. A taxonomic study on the diversity of Indian Knema Lour. (Myristicaceae). Taiwania 61(2): 141-158.

Bentham, G. \& J.D. Hooker 1883. Genera Plantarum. Vol. 3. L. Reeve \& Co., London. pp. 135-137.

Chowdhery, H.J. \& D.S. Pandey 2007. Plants of Indian Botanic Garden. Botanical Survey of India, Kolkata.

CSIR, 1956. The Wealth of India - An encyclopedia of India's Raw Material Resources. Vol. 4, F-G. Council of Scientific and Industrial Research (CSIR), New Delhi.

Deb, D.B. 1981. Flora of Tripura. Vol. 1. Deep Printers, Delhi. pp. 99-101.

Drury, C.H. 1858. The useful plants of India. William H. Allen \& Co., London. pp. 1-512.

Candolle, A.L.P.P. de 1856. Prodromus systematis naturalis regni vegetabilis. Vol. 14(1). Treuttel \& Würtz. pp. 187-208.

Gandhi, K.N. 1978. Myristicaceae. In: Saldanha, C.J. \& D.H. Nicolson (eds.), Flora of Hassan
District, Karnataka, India. Amerind Publishing Co. Pvt. Ltd., New Delhi. pp. 40-43.

Gronovius, J.F. 1755. Flora Orientalis; Sive, Recensio Plantarum, quas Leonhardus Rauwolffus, Annis 1573, 1574, \& 1575 in Syria, Arabia, Mesopotamia, Babylonia, Assyria, Armenia \& Judaea Crescentes Observavit, \& Collegit, Earumdemque Ducenta Specimena, quae in Bibliotheca Publica LugdunoBatava Adservantur, Nitidissime Exsiccata et Chartae Adglutinata in Volumen Retulit. Has Methodo Sexuali Disposuit, Synonymis Probatioribus Illustravit, Nominibusque Specificis Insignivit. Wilhelm de Groot, Leiden.

Holmgren, P., Holmgren, N. \& L. Barnett 1990. Index Herbariorum. Part I: The herbaria of the world. Eighth edition. Regnum Veg. 120. New York Botanical Garden Press, New York.

Hooker, J.D. 1886-1890. The Flora of British India. Vol. 5. L. Reeve \& Co. Ltd., London. pp. 101-114.

Hooker, J.D. \& T. Thomson 1855. Flora Indica. Vol. 1. W. Pamplin, London. pp. 153-164.

Houttuyn, M. 1773-74. Myristica fragrans Houtt. Natuurlykehistorie: of, Uitvoerigebeschryving der dieren, planten, en mineraalen 2(3). de Evren van F. Houttuyn, Te Amsterdam, p. 333.

IUCN, 2017. The IUCN Red List of Threatened Species. Version 2017-1. <http:/ / www.iucnredlist.org>. Downloaded on 28 May 2017.

King, G. 1891. The species of Myristica of British India. Ann. Roy. Bot. Gard. Calcutta 3: 286-327.

Lamarck, P.M.D. de 1791. Memoire sur le genre Du Muscadier, Myristica. Hist. Acad. Roy. Sci. (Paris): 148-168.

Ludwig, C.G. 1760. Definitiones Generum Plantarum. (edition 3; co-authored and edited by G.R. Böhmer). Ex Officina I.F. Gleditschii, Lipsiae. p. 513.

Nayar, T.S., Rasiya Beegam, A \& M. Sibi. 2014. Flowering Plants of the Western Ghats, India. Vol. 1. Jawaharlal Nehru Tropical Botanic Garden and Research Institute, Thiruvananthapuram.

Roxburgh, W. 1832. Flora Indica. Vol. 3. Serampore for W. Thacker \& Co., Calcutta \& Parbury, Allen \& Co., London..

Sinclair, J. 1958. A revision of the Malayan Myristicaceae. Gard. Bull. Singapore 16: 205-472.

Sinclair, J. 1961. The genus Knema (Myristicaceae) in Malaysia and outside Malaysia. Gard. Bull. Singapore 18: 1-327. 
Sinclair, J. 1968. The genus Myristica in Malesia and outside Malesia. Gard. Bull. Singapore 22: $1-540$.

Sinclair, J. 1975. The genus Horsfieldia (Myrisicaceae) in and outside Malesia. Gard. Bull. Singapore 28: 1-181.

Warburg, O. 1897. Monographie der Myristicaceen. Nova Acta Acad. Caes. Leop. Carol. German. Nat. Cur., 68. 1-680, tt. 1-25..

Wilde, W.J.J.O. de 1979. New account of the genus Knema (Myristicaceae). Blumea 25: 321-478.

Wilde, W.J.J.O. de 1984a. Endocomia, a new genus of Myristicaceae. Blumea 30: 173-196.
Wilde, W.J.J.O. de 1984b. A new account of the genus Horsfieldia (Myristicaceae), Pt 1. Gard. Bull. Singapore 37(2): 115-179.

Wilde, W.J.J.O. de 1997. Notes on Southeast Asian and Malesian Myristica and description of new taxa (Myristicaceae). Blumea 42: 111-190.

Wilde, W.J.J.O. de 2000. Myristicaceae. In: Stevens, P.F. (ed.), Flora Malesiana Ser. I, Vol. 14. Nationaal Herbarium Nederland, Universiteit Leiden branch, The Netherlands. pp. 1-632.

Received: 14.10 .2014

Revised and Accepted: 6.6.2017 